\title{
Closed Irrigation System for Wound Management of Complicated Ischial Pressure Ulcers
}

\author{
Kun-Yong Sung ${ }^{1}$ (D), Sang-Yeul Lee ${ }^{2}$ (D) \\ ${ }^{1}$ Department of Plastic and Reconstructive Surgery, Kangwon National University School of Medicine, Chuncheon; ${ }^{2}$ Department of Plastic and Reconstructive \\ Surgery, Kangwon National University Hospital, Chuncheon, Korea
}

\begin{abstract}
In patients with complicated ischial pressure ulcers, surgical repair is often delayed until the general condition of the patient improves, and in extremely ill patients, surgery might never be performed. Since deep ischial ulcers are usually associated with a profuse discharge, large sinus cavity, and are adjacent to the anus, maintaining effective dressing is challenging for surgeons. In order to manage such ischial ulcers, we devised a closed irrigation system using the Barovac $(50-90 \mathrm{mmHg}$ ) wound drainage device and a saline infusion set. The system was applied to a 50-year-old paraplegic man who was diagnosed with impending sepsis due to a longstanding ischial ulcer with secondary infection. Using the saline infusion set, we infused $200 \mathrm{~mL}$ of saline into the ischial cavity every 6 hours in the ward. After a 10-minute dwell time, the fluid in the cavity, including the exudate and wound debris, was drained continuously through the wound drainage device. This system was very helpful in keeping the patient's ischial wound clean until his general condition improved and surgical repair could be undertaken. We therefore believe that our closed irrigation system provides an effective, convenient, and inexpensive dressing method for patients with complicated ischial wounds.
\end{abstract}

Keywords: Ischium; Pressure ulcer; Wounds and injuries; Drainage; Suction

\section{Introduction}

Prolonged pressure on the ischium in nonambulatory patients often causes ischial ulcers. A pressure ulcer usually starts as a small superficial wound, but without adequate management, becomes deeper and eventually requires surgical repair [1]. In patients with wound infections, poor nutrition, and uncontrolled chronic diseases, surgical repair may have to be delayed until their condition improves, and surgery might never be performed in extremely ill patients. In such patients, open wounds are often challenging to manage with conventional dressing methods because they generally produce a profuse exudate, involve a large sinus cavity, and are easily contaminated from the neighboring anus. In order to effectively manage intractable ischial wounds, we devised a closed irrigation system using the Barovac (50-90 mm $\mathrm{mg}$ ) wound drainage device and a saline infusion set and applied the system to a 50-yearold paraplegic patient with a complicated ischial ulcer. This system contributed significantly to keeping the patient's ischial wound clean until his general condition improved and surgical repair could be undertaken. In this study, we describe our closed irrigation system for managing complicated ischial wounds. This study was approved by the Institutional Review Board (IRB) of Kangwon National University Hospital (IRB No. KNUH-2020-09-009-002). The patient provided written informed consent for the publication and the use of his images.

\section{Ideas and Innovations}

Received: November 10, 2020

Revised: December 15, 2020

Accepted: December 16, 2020

\section{Corresponding author:}

Sang-Yeul Lee, M.D., Ph.D.

Department of Plastic and Reconstructive Surgery, Kangwon National University Hospital, 156 Baengnyeong-ro, Chuncheon 24289, Korea

Tel: +82-33-258-9258

Fax: +82-33-258-4920

E-mail: serafin5@unitel.co.kr

This is an Open Access article distributed under the terms of the Creative Commons Attribution Non-Commercial License (https://creativecommons.org/licenses/by-nc/4.0/) which permits unrestricted non-commercial use, distribution, and reproduction in any medium, provided the original work is properly cited.

(c) 2021 Korean Wound Management Society 


\section{Ideas}

A 50-year-old paraplegic man was admitted due to a longstanding deep ischial ulcer accompanied by a high fever. The ischial open wound showed a large sinus cavity extending to the vicinity of the anus along with a profuse discharge (Fig. 1). Since the patient's vital signs and laboratory results were consistent with a clinical diagnosis of impending sepsis caused by secondary infection superimposed on the ischial ulcer, intravenous administration of vancomycin and piperacillin/tazobactam was started after performing wound and blood culture tests on the recommendation of an infectious disease specialist. On the 2nd day after admission, the ischial open wound was profusely irrigated with a saline solution in the operating room, followed by direct closure along with debridement of the margin after inserting the wound tubing of a Barovac (Sewoon Medical, Seoul, Korea) wound drainage device in the ischial cavity. The main tip of the $\mathrm{Y}$ connector at the end of the evacuating tube of the Barovac wound drainage device, after having been cut short, was connected to the rubber connector of a saline infusion set, while the side tip of the Y connector was connected to the wound tubing (Fig. 2). In the ward, 200 $\mathrm{mL}$ of saline was infused every 6 hours through the saline infusion set, and fluid in the wound cavity, including exudate and debris, was evacuated continuously through the wound drainage device after a 10-minute dwell time by nursing staff (Fig. 3). The evacuating tube was locked during saline infu-

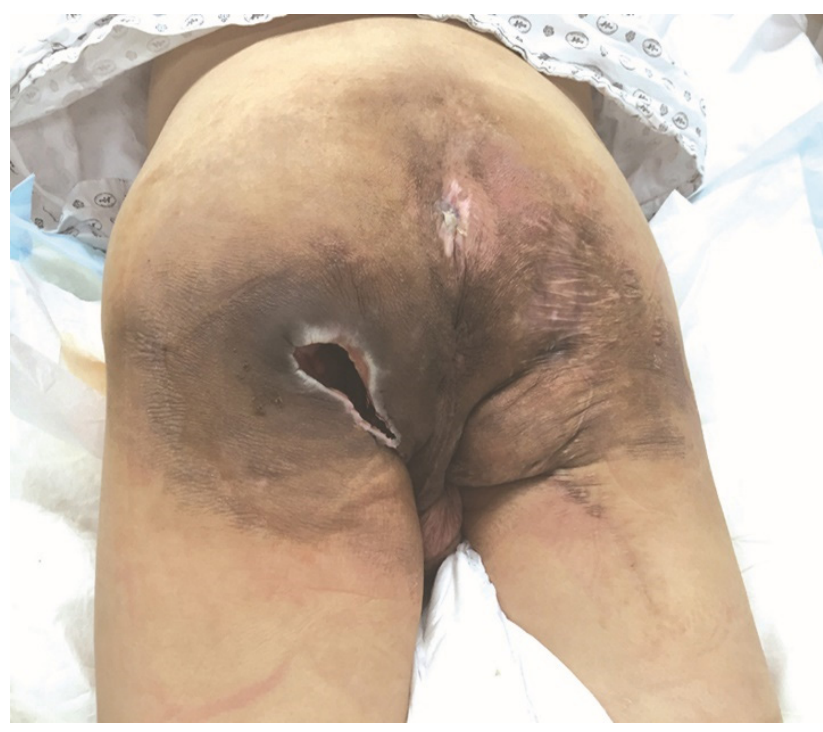

Fig. 1. A complicated ischial wound. A deep ischial ulcer on the left buttock of a 50-year-old paraplegic man. sion, and the saline infusion set was locked during evacuation. Wound management was maintained using this system until the general condition of the patient improved. Subsequently, surgical repair using the internal pudendal artery perforator flap after radical debridement and bursectomy was performed in the 2nd week.

\section{Discussion}

Wound dressing plays an important role in wound healing and management. Over the past several decades, the concept of wound dressing has changed. Today it focuses on promoting wound healing as well as wound protection. Consequently, moist dressing and negative-pressure wound therapy (NPWT) have been introduced and are widely used to manage various wounds. NPWT, also known as a vacuum-assisted closure, is an established treatment option to remove excess exudate and promote healing in acute or chronic wounds. Negative pressure is understood as aiding wound healing through a number of mechanisms including macrostrain, microstrain, fluid removal and optimizing the wound environment [2]. For man-

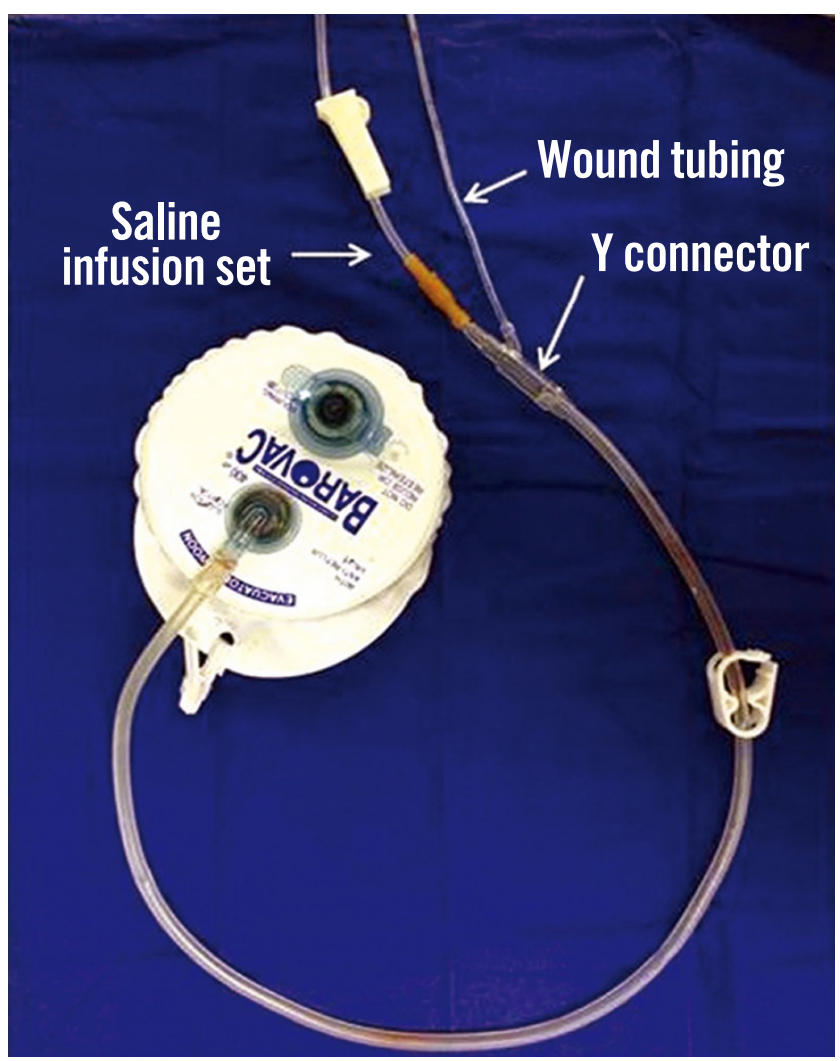

Fig. 2. A closed irrigation system. The system is composed of a Barovac wound drainage system and saline infusion set. 


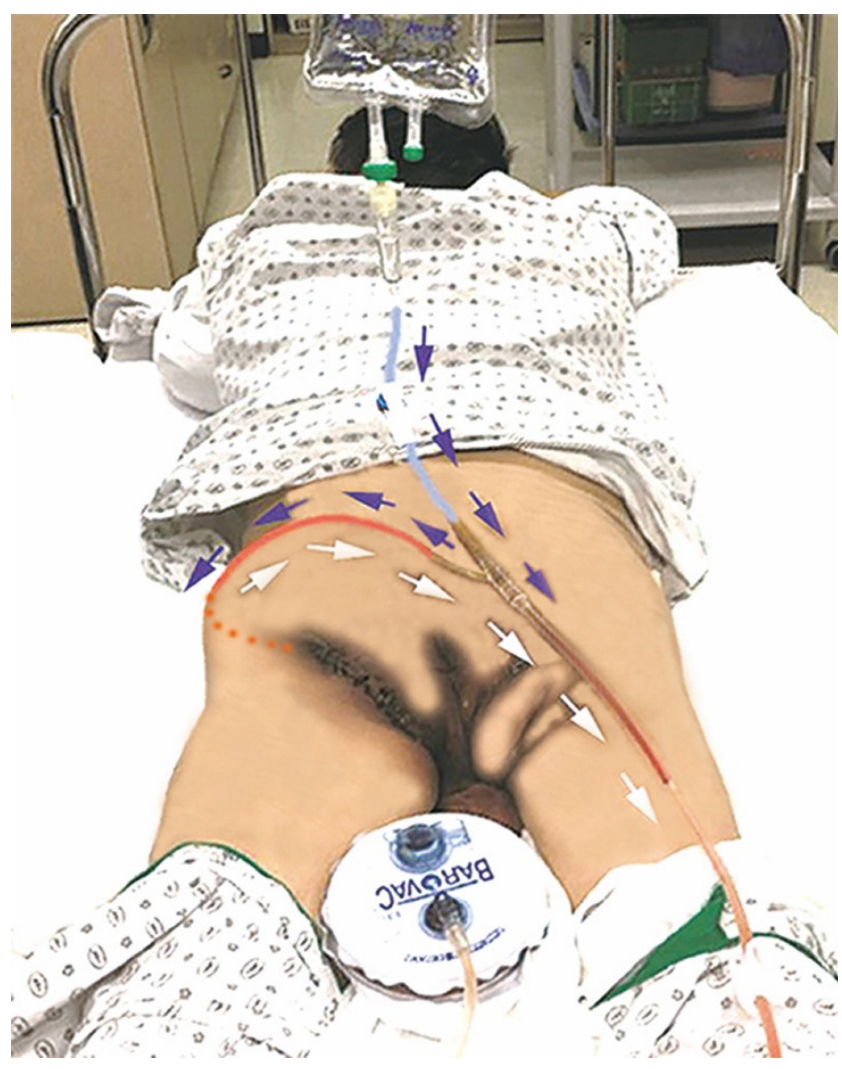

Fig. 3. Installation of the closed irrigation system in the ischial cavity. Blue arrows indicate the direction of the saline infusion and white arrows indicate the direction of drainage.

agement of complex wounds with infection, NPWT with instillation and dwell time (NPWTi-d) can be used as adjuvant therapy along with appropriate debridement and antibiotic therapy [3]. This therapy can control the range of fluid instillation and irrigation and has been reported to overcome some limitations of standard NPWT [4] by delivering topical wound solutions directly to the wound, according to conditions set by the physicians. It therefore allows the solution to dwell over the wound bed, and removes the solution during the negative pressure phase [3]. This device was proposed to enable complete wound bed preparation even in relatively severe conditions such as infected wounds or exposure of bone or tendon [4].

Wound irrigation is an essential part of wound management, and is used to remove foreign material, reduce bacterial burden, and remove cellular debris or exudate from the surface of the wound [5]. The benefits of regular wound cleansing with a topical solution in NPWT have become more apparent in recent years, especially for addressing impediments to healing [6]. Various topical solutions have been applied for this purpose; a recent study demonstrated that instilling normal saline achieved comparative outcomes as with other antiseptic solutions [7]. Other case series have also reported successful outcomes with saline $[8,9]$. While NPWTi-d can also be considered for the management of complicated ischial ulcers accompanied by profuse discharge due to secondary infection (as in our case), it is difficult to apply the dressing kit and maintain an occlusive dressing in the ischial area. Therefore, our system can be used as an alternative to NPWTi-d. We also observed that wound cleansing with the regular instillation of saline in our system contributed to better wound management.

The system we employed has several advantages over traditional wound dressing methods. Firstly, the dressing is very convenient because the open wound has already been closed. Secondly, secondary infection from fecal contamination can be prevented. Thirdly, using the proposed approach prevents the bedsheets and clothes of the patients from becoming wet. Further, our system is easily applicable and inexpensive. Although our research is insufficient for establishing guidelines for our system, we suggest a 100-200 mL saline infusion every 6 hours, followed by continuous drainage with $50-90 \mathrm{mmHg}$ of negative pressure after a 10-minute dwell time. The amount of saline infusion depends on the size of the wound cavity. Meanwhile, the instilled solution should be allowed to dwell in the wound so that it has adequate time to cleanse the wound [10]. Though appropriate instillation dwell time has been proposed at 10 minutes [6], the dwell time and intervals between saline infusion can be adjusted according to the wound conditions. Alternatively, our system can be easily modified by adding a medical three-way stopcock to the saline infusion tubing when infusion of an antiseptic solution is needed in the wound cavity.

Wound debris often causes clogging of the wound tubing. A squeezing technique or passage of a fine cannula can help clear the blockage. For intractable blockages, the wound tubing can be easily replaced with new tubing through a small incision in the previous wound closure site. When replacing old tubing, microbial culture for its tip may be considered.

Our system has limited indications; in particular, it should only be applied to open wounds that can be closed directly. Nevertheless, we argue that our closed irrigation system using the Barovac wound drainage device and a saline infusion set can help keep wounds clean and prevent secondary infection not only in patients with complicated ischial ulcers, but also in chronically debilitated patients with irreparable ischial sores. 


\section{Conflict of interest}

Kun-Yong Sung is an editorial board member of the journal but did not involve in the peer reviewer selection, evaluation, or decision process of this article. No other potential conflicts of interest relevant to this article were reported.

\section{ORCID iDs}

Kun-Yong Sung

https://orcid.org/0000-0002-3114-0001

Sang-Yeul Lee

https://orcid.org/0000-0001-5262-1499

\section{References}

1. Sorensen JL, Jorgensen B, Gottrup F. Surgical treatment of pressure ulcers. Am J Surg 2004;188(1A Suppl):42-51.

2. Huang C, Leavitt T, Bayer LR, et al. Effect of negative pressure wound therapy on wound healing. Curr Probl Surg 2014; 51:301-31.

3. Anghel EL, Kim PJ, Attinger CE. A solution for complex wounds: the evidence for negative pressure wound therapy with instillation. Int Wound J 2016;13Suppl 3:19-24.
4. Nishii Y. Clinical experience of negative pressure wound therapy with instillation. J Wound Manag Res 2019;15:3-4.

5. Lewis K, Pay JL. Wound irrigation [Internet]. Treasure Island: StatPearls Publishing; c2019 [cited 2021 Jan 31]. Available from: https://www.ncbi.nlm.nih.gov/books/NBK538522/.

6. Kim PJ, Attinger CE, Constantine T, et al. Negative pressure wound therapy with instillation: international consensus guidelines update. Int Wound J 2020;17:174-86.

7. Kim PJ, Attinger CE, Oliver N, et al. Comparison of outcomes for normal saline and an antiseptic solution for negative-pressure wound therapy with instillation. Plast Reconstr Surg 2015;136:657e-664e.

8. Fluieraru S, Bekara F, Naud M, et al. Sterile-water negative pressure instillation therapy for complex wounds and NPWT failures. J Wound Care 2013;22:293-9.

9. Brinkert D, Ali M, Naud M, et al. Negative pressure wound therapy with saline instillation: 131 patient case series. Int Wound J 2013;10 Suppl 1:56-60.

10. Willy C, Scheuermann-Poley C, Stichling M, et al. Importance of wound irrigation solutions and fluids with antiseptic effects in therapy and prophylaxis: update 2017. Unfallchirurg 2017;120:549-60. 